\title{
A MAXIMUM PRINCIPLE FOR OPTIMAL CONTROL FOR A CLASS OF CONTROLLED SYSTEMS
}

\author{
WENSHENG $\mathrm{XU}^{1}$
}

(Received 1 July 1994; revised 28 February 1995)

\begin{abstract}
Applying Ekeland's variational principle in this paper, we obtain a maximum principle for optimal control for a class of two-point boundary value controlled systems. The control domain need not be convex. For a special case, that is the so called LQ-type problem, we obtain the optimal control in the closed loop form and a corresponding Riccati type differential equation.
\end{abstract}

\section{Introduction}

In this paper we discuss an optimal control problem: minimize the cost function

$$
J(v(\cdot), x(0))=h(x(0))+\gamma(x(T))
$$

subject to the system

$$
\dot{x}(t)=f(x(t), v(t))
$$

with initial and terminal state constraints $G_{0}(x(0))=0$ and $G_{1}(x(T))=0$ respectively, where $v(\cdot)$ is the control function mapping into a subset of $R^{k}$ and $x(\cdot)$ is the state of the system. We call the above system a two-point boundary value controlled system. Our objective is to obtain a necessary condition, called a maximum principle, for an optimal control of the problem.

The paper is motivated by Pontryagin et al. [4]. Applying a convex cone method in the celebrated book, Pontryagin discussed an optimal control problem with initial and endpoint state constraints. In this paper we obtain a maximum principle by using Ekeland's variational principle [3], where the transversality conditions are in a more precise form. (See (14) and (16) for details.)

\footnotetext{
${ }^{1}$ Department of Mathematics, Zhejiang University, Hangzhou 310027, P. R. China.

(C) Australian Mathematical Society, 1996, Serial-fee code 0334-2700/96
} 
We may sometimes allow the initial state to vary in some given range. Rehbock et al. [5] studied the sensitivity of the optimum cost with respect to changes in the coefficient vector, especially in the initial state of the dynamical system. They formulated a new cost function by penalizing the sensitivity of the original objective functional and solved the problem by using the control parameterization technique [6]. See [5] for further details.

Only necessary conditions for the optimal control are discussed. We do not incorporate the existence problem. Some results in this field can be found in Ahmed [1, 2].

The problem is stated in detail in Section 2 and the maximum principle derived for the optimal control in Section 3. In Section 4, we consider a specific LQ-type problem and obtain the optimal control in the closed loop form and a corresponding Riccati type differential equation. We give a brief conclusion in the last section.

\section{Statement of the problem}

Let $f, G_{0}, G_{1}, h$ and $\gamma$ be given maps such that $f: R^{n} \times R^{k} \rightarrow R^{n} ; G_{0}: R^{n} \rightarrow$ $R^{n_{0}}, n_{0}<n ; G_{1}: R^{n} \rightarrow R^{n_{1}}, n_{1}<n ; h: R^{n} \rightarrow R^{1}$ and $\gamma: R^{n} \rightarrow R^{1}$. We assume

(H1) $f$ is continuous with respect to $(x, v)$ and continuously differentiable with respect to $x$;

(H2) $G_{0}, G_{1}, h$ and $\gamma$ are continuously differentiable.

Let $U$ be a nonempty closed subset of $R^{k}$. Define the set $\mathscr{U}_{a d}$ of admissable controls as

$$
\mathscr{U}_{a d}=\left\{v(\cdot) \in L^{\infty}\left(0, T ; R^{k}\right): v(t) \in U, \quad \text { a.e. } t \in[0, T]\right\},
$$

where $L^{\infty}\left(0, T ; R^{k}\right)$ denotes the Banach space of all essentially bounded measurable functions from $[0, T]$ to $R^{k}$.

We consider the controlled system

$$
\dot{x}(t)=f(x(t), v(t)), \quad G_{0}(x(0))=0, G_{1}(x(T))=0,
$$

where $G_{0}(x(0))=0$ and $G_{1}(x(T))=0$ are initial and terminal state constraints respectively. For any $x(0) \in\left\{x: G_{0}(x)=0\right\}$ and $v(\cdot) \in \mathscr{U}_{a d}$, the solution $x(\cdot)=$ $x(\cdot ; v(\cdot), x(0))$ of the system $(1)$ is called a trajectory corresponding to $(v(\cdot), x(0))$ if it satisfies the terminal state constraints. For any $x(0) \in\left\{x: G_{0}(x)=0\right\}$ and $v(\cdot) \in \mathscr{U}_{a d}$, define the cost function

$J(v(\cdot), x(0))= \begin{cases}h(x(0))+\gamma(x(T ; v(\cdot), x(0)), & \text { if the trajectory } x(\cdot ; v(\cdot), x(0)) \text { exists, } \\ +\infty, & \text { otherwise. }\end{cases}$ 
The optimal control problem is to find a pair $(v(\cdot), x(0)) \in \mathscr{U}_{a d} \times\left\{x: G_{0}(x)=0\right\}$ minimizing the cost function (2).

If $\left(u(\cdot), x_{0}\right) \in \mathscr{U}_{a d} \times\left\{x: G_{0}(x)=0\right\}$ attains the minimum of $J(v(\cdot), x(0)), x_{0}$ is called an optimal initial state and $u(\cdot)$ is called an optimal control corresponding to $x_{0}$.

\section{The maximum principle}

In this section, we first introduce the variational equation and derive the variational inequality. Then we apply Ekeland's principle to obtain the maximum principle.

Let $\left(u(\cdot), x_{0}, x(\cdot)\right)$ be an optimal solution to the optimal control problem (1) and (2). Define the variational control

$$
u^{\epsilon}(t)= \begin{cases}v, & \tau \leq t \leq \tau+\epsilon, \\ u(t), & \text { otherwise, }\end{cases}
$$

where $v \in U, \tau \in[0, T)$ and $\epsilon>0$ is sufficiently small.

Let us consider a variational form of (1) with initial state perturbation

$$
\dot{x}(t)=f(x(t), v(t)), \quad x(0)=x_{0}+\xi,
$$

where $\xi \in R^{n}$ and $v(\cdot) \in \mathscr{U}_{a d}$. Denote the solution of (3) as $x(t ; v(\cdot), \xi)$ and $x^{\epsilon}(\cdot) \triangleq x\left(\cdot ; u^{\epsilon}(\cdot), \epsilon \xi\right)$ with an arbitrarily given $\xi \in R^{n}$. For convenience, we use the following notation:

$$
f(u)=f(x(t), u(t)), \quad f\left(u^{\epsilon}\right)=f\left(x(t), u^{\epsilon}(t)\right),
$$

and introducing the variational equation

$$
\begin{aligned}
\dot{x}_{1} & =f_{x}(u) x_{1}+f\left(u^{\epsilon}\right)-f(u), \\
x_{1}(0) & =\epsilon \xi,
\end{aligned}
$$

we have the following result.

LEMMA 1. Suppose (H1) holds. For $x_{1}$, we have the estimation

$$
x^{\epsilon}(t)=x(t)+x_{1}(t)+o(\epsilon), \quad \forall t \in[0, T] .
$$

PROOF. From (3) and (4)

$$
\begin{aligned}
x^{\epsilon}(t)-x(t)-x_{1}(t) & =\int_{0}^{t}\left[f\left(x^{\epsilon}, u^{\epsilon}\right)-f\left(u^{\epsilon}\right)-f_{x}(u) x_{1}\right] d s \\
& =\int_{0}^{t}\left[\int_{0}^{1} f_{x}\left(x+\lambda\left(x^{\epsilon}-x\right), u^{\epsilon}\right) d \lambda\left(x^{\epsilon}-x\right)-f_{x}(u) x_{1}\right] d s .
\end{aligned}
$$


It follows that

$$
\begin{aligned}
\mid x^{\epsilon}(t) & -x(t)-x_{1}(t) \mid \\
& \leq \int_{0}^{t}\left|f_{x}(u)\right| \cdot\left|x^{\epsilon}(s)-x(s)-x_{1}(s)\right| d s+\left|\int_{0}^{t} A^{\epsilon} \cdot\left(x^{\epsilon}(s)-x(s)\right) d s\right|,
\end{aligned}
$$

with

$$
A^{\epsilon}=\int_{0}^{1}\left(f_{x}\left(x+\lambda\left(x^{\epsilon}-x\right), u^{\epsilon}\right)-f_{x}(x, u)\right) d \lambda
$$

One can prove that $x^{\epsilon}(t)-x(t)=O(\epsilon), \forall t \in[0, T]$. Applying Gronwall's inequality (see [7]) to the above relation, we have

$$
\left|x^{\epsilon}(t)-x(t)-x_{1}(t)\right| \leq C \cdot\left|\int_{0}^{t} A^{\epsilon}\left(x^{\epsilon}(s)-x(s)\right) d s\right|=o(\epsilon), \quad \forall t \in[0, T]
$$

with $C=e^{\int_{0}^{T}\left|f_{x}(u)\right| d t}$. This completes the proof.

We apply Ekeland's variational principle to solve our problem. Define a metric in $\mathscr{U}_{\text {ad }} \times R^{n} ;$ for $\left(v_{1}(\cdot), \xi\right),\left(v_{2}(\cdot), \eta\right) \in \mathscr{U}_{a d} \times R^{n}$, let

$$
d\left(\left(v_{1}(\cdot), \xi\right),\left(v_{2}(\cdot), \eta\right)\right) \triangleq \operatorname{meas}\{t \in[0, T]: u(t) \neq v(t)\}+\left\{\sum_{i=1}^{n}\left(\xi_{i}-\eta_{i}\right)^{2}\right\}^{1 / 2}
$$

where $\xi=\left(\xi_{1}, \cdots, \xi_{n}\right)^{T} \in R^{n}, \eta=\left(\eta_{1}, \cdots, \eta_{n}\right)^{T} \in R^{n}$ and meas is the Lebesgue measure. We can follow Ekeland [3] to prove that $\left(\mathscr{U}_{\text {ad }} \times R^{n}, d(\cdot, \cdot)\right)$ is a complete metric space.

For $(v(\cdot), \xi) \in \mathscr{U}_{a d} \times R^{n}$, consider the following cost function

$$
\begin{aligned}
F_{\epsilon}(v(\cdot), \xi)= & \left\{\left|G_{0}\left(x_{0}+\xi\right)\right|^{2}+\left|G_{1}(x(T ; v(\cdot), \xi))\right|^{2}\right. \\
& \left.+\left(h\left(x_{0}+\xi\right)+\gamma(x(T ; v(\cdot), \xi))-h(x(0))-\gamma(x(T))+\epsilon\right)^{2}\right\}^{1 / 2} .
\end{aligned}
$$

It can be proved that $F_{\epsilon}: \mathscr{U}_{a d} \times R^{n} \rightarrow R^{1}$ is continuous and

$$
F_{\epsilon}(v(\cdot), \xi) \geq 0, \quad F_{\epsilon}(u(\cdot), 0)=\epsilon .
$$

Obviously

$$
F_{\epsilon}(u(\cdot), 0) \leq \inf _{(v(\cdot), \xi) \in \mathscr{U}_{a d} \times R^{n}} F_{\epsilon}(v(\cdot), \xi)+\epsilon
$$


From Ekeland's variational principle, there exists $\left(u_{\epsilon}(\cdot), \xi_{\epsilon}\right) \in \mathscr{U}_{a d} \times R^{n}$ such that

$$
\begin{aligned}
\text { (i) } & F_{\epsilon}\left(u_{\epsilon}(\cdot), \xi_{\epsilon}\right) \leq F_{\epsilon}(u(\cdot), 0)=\epsilon \\
\text { (ii) } & d\left(\left(u_{\epsilon}(\cdot), \xi_{\epsilon}\right),(u(\cdot), 0)\right) \leq \sqrt{\epsilon} \\
\text { (iii) } & F_{\epsilon}(w(\cdot), \xi) \geq F_{\epsilon}\left(u_{\epsilon}(\cdot), \xi_{\epsilon}\right)-\sqrt{\epsilon} d\left((w(\cdot), \xi),\left(u_{\epsilon}(\cdot), \xi_{\epsilon}\right)\right), \\
& \forall(w(\cdot), \xi) \in \mathscr{U}_{a d} \times R^{n} .
\end{aligned}
$$

Define a spike variation

$$
u_{\epsilon}^{\rho}(t)= \begin{cases}v, & \tau \leq t \leq \tau+\rho, \\ u_{\epsilon}(t), & \text { otherwise, }\end{cases}
$$

with $v \in U$ and an initial state perturbation $\xi_{\epsilon}^{\rho}=\xi_{\epsilon}+\rho \xi_{0}$ with an arbitrarily given $\xi_{0} \in R^{n}$. It can be seen that $\left(u_{\epsilon}^{\rho}(\cdot), \xi_{\epsilon}^{\rho}\right) \in \mathscr{U}_{a d} \times R^{n}$ and

$$
d\left(\left(u_{\epsilon}^{\rho}(\cdot), \xi_{\epsilon}^{\rho}\right),\left(u_{\epsilon}(\cdot), \xi_{\epsilon}\right)\right) \leq\left(1+\left|\xi_{0}\right|\right) \rho
$$

where $|\cdot|$ represents the Euclidean norm on $R^{n}$.

Then it follows from (7)(iii) that

$$
F_{\epsilon}\left(u_{\epsilon}^{\rho}(\cdot), \xi_{\epsilon}^{\rho}\right)-F_{\epsilon}\left(u_{\epsilon}(\cdot), \xi_{\epsilon}\right)+\sqrt{\epsilon}\left(1+\left|\xi_{0}\right|\right) \rho \geq 0 .
$$

For notational simplification, denote $x_{\epsilon}^{\rho}(t) \triangleq x\left(t ; u_{\epsilon}^{\rho}(\cdot), \xi_{\epsilon}^{\rho}\right)$ and $x_{\epsilon}(t) \triangleq x\left(t ; u_{\epsilon}(\cdot)\right.$, $\left.\xi_{\epsilon}\right)$. Let $x_{1 \epsilon}(t)$ be the solution of

$$
\begin{aligned}
\dot{x}_{1 \epsilon} & =f_{x}\left(x_{\epsilon}, u_{\epsilon}\right) x_{1 \epsilon}+f\left(x_{\epsilon}, u_{\epsilon}^{\rho}\right)-f\left(x_{\epsilon}, u_{\epsilon}\right), \\
x_{1 \epsilon}(0) & =\rho \xi_{0} .
\end{aligned}
$$

The estimation

$$
x_{\epsilon}^{\rho}(t)=x_{\epsilon}(t)+x_{1 \epsilon}(t)+o(\rho), \quad \forall t \in[0, T]
$$

is obtained from Lemma 1. From the assumption (H2) and (9), we can derive that

$$
\begin{aligned}
\left(F_{\epsilon}\left(u_{\epsilon}^{\rho}(\cdot), \xi_{\epsilon}^{\rho}\right)\right)^{2}-\left(F_{\epsilon}\left(u_{\epsilon}(\cdot), \xi_{\epsilon}\right)\right)^{2} & \\
= & 2\left\langle G_{0 x}\left(x_{0}+\xi_{\epsilon}\right) \rho \xi_{0}, G_{0}\left(x_{0}+\xi_{\epsilon}\right)\right\rangle+2\left\langle G_{1 x}\left(x_{\epsilon}(T)\right) x_{1 \epsilon}(T), G_{1}\left(x_{\epsilon}(T)\right)\right\rangle \\
& +2\left\langle h_{x}\left(x_{0}+\xi_{\epsilon}\right) \rho \xi_{0}+\gamma_{x}\left(x_{\epsilon}(T)\right) x_{1 \epsilon}(T)\right. \\
& \left.\quad h\left(x_{0}+\xi_{\epsilon}\right)+\gamma\left(x_{\epsilon}(T)\right)-h(x(0))-\gamma(x(T))+\epsilon\right\rangle+o(\rho) .
\end{aligned}
$$

Since

$$
F_{\epsilon}\left(u_{\epsilon}^{\rho}(\cdot), \xi_{\epsilon}^{\rho}\right) \rightarrow F_{\epsilon}\left(u_{\epsilon}(\cdot), \xi_{\epsilon}\right) \quad \text { and } \quad F_{\epsilon}\left(u_{\epsilon}(\cdot), \xi_{\epsilon}\right)>0
$$


it follows from (8) and (10) that

$$
\begin{aligned}
& \left\langle G_{0 x}\left(x_{0}+\xi_{\epsilon}\right) \rho \xi_{0}, h_{1 \epsilon}\right\rangle+\left\langle G_{1 x}\left(x_{\epsilon}(T)\right) x_{1 \epsilon}(T), h_{2 \epsilon}\right\rangle+ \\
& \quad\left\langle h_{x}\left(x_{0}+\xi_{\epsilon}\right) \rho \xi_{0}+\gamma_{x}\left(x_{\epsilon}(T)\right) x_{1 \epsilon}(T), h_{0 \epsilon}\right\rangle+\rho \sqrt{\epsilon}\left(1+\left|\xi_{0}\right|\right)+o(\rho) \geq 0
\end{aligned}
$$

with

$$
\begin{aligned}
h_{0 \epsilon} & =\frac{h\left(x_{0}+\xi_{\epsilon}\right)+\gamma\left(x_{\epsilon}(T)\right)-h\left(x_{0}\right)-\gamma(x(T))+\epsilon}{F_{\epsilon}\left(u_{\epsilon}(\cdot), \xi_{\epsilon}\right)} \\
h_{1 \epsilon} & =\frac{G_{0}\left(x_{0}+\xi_{\epsilon}\right)}{F_{\epsilon}\left(u_{\epsilon}(\cdot), \xi_{\epsilon}\right)} \\
h_{2 \epsilon} & =\frac{G_{1}\left(x_{\epsilon}(T)\right)}{F_{\epsilon}\left(u_{\epsilon}(\cdot), \xi_{\epsilon}\right)}
\end{aligned}
$$

Let $p_{\epsilon}$ be the solution of

$$
\begin{aligned}
-\dot{p}_{\epsilon} & =f_{x}^{*}\left(x_{\epsilon}, u_{\epsilon}\right) \cdot p_{\epsilon}, \\
p_{\epsilon}(T) & =G_{1 x}^{*}\left(x_{\epsilon}(T)\right) h_{2 \epsilon}+\gamma_{x}^{*}\left(x_{\epsilon}(T)\right) h_{0 \epsilon} .
\end{aligned}
$$

Then the inequality

$$
\begin{aligned}
& \left\langle p_{\epsilon}(0)+G_{0 x}^{*}\left(x_{0}+\xi_{\epsilon}\right) \cdot h_{1 \epsilon}+h_{x}^{*}\left(x_{0}+\xi_{\epsilon}\right) \cdot h_{0 \epsilon}, \rho \xi_{0}\right\rangle \\
& \quad+\int_{0}^{T}\left[H\left(x_{\epsilon}, u_{\epsilon}^{\rho}, p_{\epsilon}\right)-H\left(x_{\epsilon}, u_{\epsilon}, p_{\epsilon}\right)\right] d t+\rho \sqrt{\epsilon}\left(1+\left|\xi_{0}\right|\right)+o(\rho) \geq 0
\end{aligned}
$$

follows from (11), where $H(x, v, p) \triangleq\langle p, f(x, v)\rangle$ is the Hamiltonian function.

Multiplying both sides of (12) by $1 / \rho$ and then letting $\rho \rightarrow 0$, yields

$$
\begin{aligned}
& \left\langle p_{\epsilon}(0)+G_{0 x}^{*}\left(x_{0}+\xi_{\epsilon}\right) \cdot h_{1 \epsilon}+h_{x}^{*}\left(x_{0}+\xi_{\epsilon}\right) \cdot h_{0 \epsilon}, \xi_{0}\right\rangle \\
& \quad+H\left(x_{\epsilon}, v, p_{\epsilon}\right)-H\left(x_{\epsilon}, u_{\epsilon}, p_{\epsilon}\right)+\sqrt{\epsilon}\left(1+\left|\xi_{0}\right|\right) \geq 0 .
\end{aligned}
$$

Since $\sum_{i=0}^{2}\left|h_{i \epsilon}\right|^{2}=1$, there exists a convergent subsequence of $\left\{h_{i \epsilon}\right\}$ such that

$$
h_{i \epsilon} \rightarrow h_{i}, \quad \epsilon \rightarrow 0, \quad i=0,1,2
$$

From (7)(ii), it follows that $\left(u_{\epsilon}(\cdot), \xi_{\epsilon}\right) \rightarrow(u(\cdot), 0)$ in $\left(\mathscr{U}_{a d} \times R^{n}, d\right)$ and $x_{\epsilon}(t) \rightarrow$ $x(t), p_{\epsilon}(t) \rightarrow p(t), \epsilon \rightarrow 0, \forall t \in[0, T]$, where $p(\cdot)$ is the solution of the following adjoint equation

$$
\begin{aligned}
-\dot{p} & =f_{x}^{*}(x, u) \cdot p \\
p(T) & =G_{1 x}^{*}(x(T)) \cdot h_{2}+\gamma_{x}^{*}(x(T)) \cdot h_{0}
\end{aligned}
$$


Letting $\epsilon \rightarrow 0$ in (13), yields

$$
\left\langle p(0)+G_{0 x}^{*}\left(x_{0}\right) \cdot h_{1}+h_{x}^{*}\left(x_{0}\right) \cdot h_{0}, \xi_{0}\right\rangle+H(x, v, p)-H(x, u, p) \geq 0
$$

and since $\xi_{0} \in R^{n}$ is arbitrary

$$
H(x(t), v, p(t)) \geq H(x(t), u(t), p(t)), \quad \forall v \in U, \quad \text { a.e. } t \in[0, T],
$$

and

$$
p(0)=-\left(G_{0 x}^{*}\left(x_{0}\right) \cdot h_{1}+h_{x}^{*}\left(x_{0}\right) \cdot h_{0}\right) .
$$

THEOREM 1. Suppose $(\mathrm{H} 1)$ and $(\mathrm{H} 2)$ hold. Let $\left(u(\cdot), x_{0}, x(\cdot)\right)$ be an optimal solution to the problem (1) and (2), and $p(\cdot)$ the corresponding solution of the adjoint equation (14) and (16). Then the maximum principle (15) holds.

\section{A LQ-type optimal control problem}

In this section, we discuss a specific LQ-type optimal control problem. First consider two special cases of (1) and (2).

CASE 1. Minimize the cost function

$$
J(v(\cdot))=h(y(0))+\gamma(x(T))
$$

subject to the controlled system

$$
\begin{array}{ll}
\dot{x}=f(x, v), & x(0)=x_{0}, \\
\dot{y}=g(x, y, v), & y(T)=m(x(T)),
\end{array}
$$

where $v(\cdot) \in \mathscr{U}_{a d}, f: R^{n} \times R^{k} \rightarrow R^{n}, g: R^{n} \times R^{m} \times R^{k} \rightarrow R^{m}, m: R^{n} \rightarrow R^{m}$, $h: R^{m} \rightarrow R^{1}$ and $\gamma: R^{n} \rightarrow R^{1}$. Assume (H1), (H2) and an additional condition

(H3) $g$ is continuous with respect to $(x, y, v)$ and continuously differentiable with respect to $x, y ; m$ is continuously differentiable.

The following result follows naturally from Theorem 1 .

COROLlaRY 1. Süpose (H1), (H2) and (H3) hold. Let $(u(\cdot),(x(\cdot), y(\cdot))$ be an optimal solution to the problem (17) and (18), and let $(p(\cdot), q(\cdot))$ be the corresponding solution of the adjoint equation

$$
\left\{\begin{aligned}
-\dot{p} & =f_{x}^{*}(x, u) \cdot p+g_{x}^{*}(x, y, u) \cdot q, \\
p(T) & =\gamma_{x}(x(T))-m_{x}^{*}(x(T)) q(T), \\
-\dot{q} & =g_{y}^{*}(x, y, u) \cdot q, \\
q(0) & =-h_{y}(y(0)) .
\end{aligned}\right.
$$


Then the maximum principle

$$
H(x(t), y(t), v, p(t), q(t)) \geq H(x(t), y(t), u(t), p(t), q(t)), \forall v \in U, \text { a.e. } t,
$$

holds, where $H(x, y, v, p, q) \triangleq\langle p, f(x, v)\rangle+\langle q, g(x, y, v)\rangle$ is the Hamiltonian function.

CASE 2. Minimize the cost function

$$
J(v(\cdot))=h(y(0))+\gamma(x(T))+\int_{0}^{T} l(x, y, v) d t,
$$

subject to system (18). Assume that $l(x, y, v)$ satisfies the conditions analoguous to that $g(x, y, v)$ satisfies in $(\mathrm{H} 3)$.

Defining a new state $z(t)=\int_{t}^{T} l(x, y, v) d s$ it is easily seen that the problem is in the form of Case 1 . We have the following result from Corollary 1.

COROLLARY 2. Suppose all the assumptions hold. Let $(u(\cdot),(x(\cdot), y(\cdot))$ be an optimal solution to the problem (21) and (18), and let $(p(\cdot), q(\cdot))$ be the corresponding solution of the adjoint equation

$$
\left\{\begin{aligned}
-\dot{p} & =f_{x}^{*}(x, u) \cdot p+g_{x}^{*}(x, y, u) \cdot q+l_{x}(x, y, u), \\
p(T) & =\gamma_{x}(x(T))-m_{x}^{*}(x(T)) \cdot q(T), \\
-\dot{q} & =g_{y}^{*}(x, y, u) \cdot q+l_{y}(x, y, u), \\
q(0) & =-h_{y}(y(0)) .
\end{aligned}\right.
$$

Then the maximum principle (20) holds, where the Hamiltonian function is

$$
H(x, y, v, p, q) \triangleq\langle p, f(x, v)\rangle+\langle q, g(x, y, v)\rangle+l(x, y, v) .
$$

Let us now consider a LQ-type optimal control problem. The linear controlled system is

$$
\begin{array}{ll}
\dot{x}=A x+B v, & x(0)=x_{0}, \\
\dot{y}=C x+D y+E v, & y(T)=W x(T),
\end{array}
$$

where $v(\cdot) \in \mathscr{U}_{a d}, U=R^{k}, x \in R^{n}$ and $y \in R^{m}$. The objective is to find a $u(\cdot) \in \mathscr{U}_{a d}$ minimizing the quadratic cost function

$$
\begin{aligned}
J(v(\cdot))= & \frac{1}{2} x^{*}(T) W_{1} x(T)+\frac{1}{2} y^{*}(0) W_{2} y(0) \\
& +\frac{1}{2} \int_{0}^{T}\left[u^{*}(t) R u(t)+x^{*}(t) Q_{1} x(t)+y^{*}(t) Q_{2} y(t)\right] d t,
\end{aligned}
$$


where $A \in R^{n \times n}, B \in R^{n \times k}, C \in R^{m \times n}, D \in R^{m \times m}, E \in R^{m \times k}, W \in R^{m \times n}$, $W_{1} \in R^{n \times n}, W_{2} \in R^{m \times m}, R \in R^{k \times k}, Q_{1} \in R^{n \times n}$ and $Q_{2} \in R^{m \times m}$ are matrices with

$$
R>0, \quad W_{1} \geq 0, \quad W_{2} \geq 0, \quad Q_{1} \geq 0, \quad Q_{2} \geq 0 .
$$

Obviously, this problem is in the form of Case 2 . Corollary 2 gives the optimal control and the adjoint equation in the following form,

$$
u=-R^{-1}\left(B^{*} p+E^{*} q\right),
$$

and

$$
\begin{array}{ll}
-\dot{p}=A^{*} p+C^{*} q+Q_{1} x, & p(T)=W_{1} x(T)-W q(T), \\
-\dot{q}=D^{*} q+Q_{2} y, & q(0)=-W_{2} y(0) .
\end{array}
$$

Moreover, the optimal trajectory is the solution of the following system

$$
\begin{array}{ll}
\dot{x}=A x-B R^{-1}\left(B^{*} p+E^{*} q\right), & x(0)=x_{0}, \\
\dot{y}=C x+D y-E R^{-1}\left(B^{*} p+E^{*} q\right), & y(T)=W x(T) .
\end{array}
$$

Now we try to solve the dual equations (26) and (27). Letting $p(t)=F_{1}(t) x(t)$, $q(t)=F_{2}(t) x(t)$ and $y(t)=F_{3}(t) x(t)$, one can easily show that $F_{1}(t), F_{2}(t)$ and $F_{3}(t)$ satisfy the following Riccati type differential equations

$$
\begin{gathered}
\dot{F}_{1}+F_{1} A+A^{*} F_{1}-F_{1} B R^{-1}\left(B^{*} F_{1}+E^{*} F_{2}\right)+C^{*} F_{2}+Q_{1}=0 \\
F_{1}(T)=W_{1}-W F_{2}(T), \\
\dot{F}_{2}+F_{2} A+D^{*} F_{2}-F_{2} B R^{-1}\left(B^{*} F_{1}+E^{*} F_{2}\right)+Q_{2} F_{3}=0, \\
F_{2}(0)=-W_{2} F_{3}(0) \\
\dot{F}_{3}+F_{3} A-D F_{3}-\left(F_{3} B-E\right) R^{-1}\left(B^{*} F_{1}+E^{*} F_{2}\right)-C=0 \\
F_{3}(T)=W .
\end{gathered}
$$

From (25) and (28), the optimal control is in the closed loop form

$$
u(t)=-R^{-1}\left(B^{*} F_{1}(t)+E^{*} F_{2}(t)\right) x(t),
$$

where $x(\cdot)$ is the corresponding optimal trajectory.

THEOREM 2. Let $(u(\cdot), x(\cdot), y(\cdot))$ be an optimal solution to the LQ-type problem (23) and (24). Then, if the corresponding Riccati type equation (28) has some solution $\left(F_{1}(\cdot), F_{2}(\cdot), F_{3}(\cdot)\right)$, the optimal control $u(\cdot)$ is in the closed loop form (29). 


\section{Conclusion}

In this paper, we consider a class of optimal control problem (1) and (2) in which the controlled dynamical system (1) is a two-point boundary value system. The objective is to find a control which minimizes the cost function (2), where the initial state of the system is treated as controls. To solve the problem, we apply Ekeland's variational principle. The necessary condition (15) for the optimal control is derived. We note that the corresponding adjoint equations (14) and (16) are a two-point boundary value system. For the specific LQ-type problem (23) and (24), we obtain the optimal control (29) in the closed loop form.

\section{Acknowledgements}

The author thanks Professor Shige Peng for some helpful advice on the paper. The author also thanks the associate editor and the anonymous referees for suggesting valuable improvements in the presentation. This work was supported by the National Natural Science Foundation of China.

\section{References}

[1] N. U. Ahmed, "Existence of optimal controls for a class of systems governed by differential inclusions on a Banach space", J. Optim. Theory Appl. 50 (1986) 213-237.

[2] N. U. Ahmed, Elements of finite-dimensional systems and control theory (Longman, England, 1988).

[3] I. Ekeland, "On the variational principle", J. Math. Anal. and Applic. 47 (1974) 324-353.

[4] L. S. Pontryagin et al., The Mathematical Theory of Optimal Processes (Wiley, New York, 1962).

[5] V. Rehbock, K. L. Teo and L. S. Jennings, "A computational procedure for suboptimal robust controls", Dynamics and Control 2 (1992) 331-348.

[6] K. L. Teo, C. J. Goh and K. H. Wong, A unified computational approach to optimal control problems (Longman, England, 1991).

[7] R. Wang and Z. Wu, Ordinary differential equations, in Chinese (Advanced Educational Publishing Company, Peking, 1987). 